\title{
Adaptación y validación del instrumento de calidad de vida Diabetes 39 en pacientes mexicanos con diabetes mellitus tipo 2
}

\author{
Juan Manuel López-Carmona, MIC,(1) Raymundo Rodríguez-Moctezuma, MIC.(2)
}

\author{
López-Carmona JM, Rodríguez-Moctezuma $\mathbf{R}$. \\ Adaptación y validación del instrumento de calidad \\ de vida Diabetes 39 en pacientes mexicanos \\ con diabetes mellitus tipo 2. \\ Salud Publica Mex 2006;48:200-2II.
}

\section{Resumen}

Objetivo.Adaptar y validar la versión al español del cuestionario Diabetes 39, que mide calidad de vida, en pacientes mexicanos con diabetes mellitus tipo 2 (DM-2). Material y métodos. Encuesta transversal realizada en una unidad de medicina familiar. Un panel de investigadores adaptó el instrumento, que se aplicó en dos ocasiones, por autoadministración, a una muestra de 260 pacientes con DM-2. Se midieron la hemoglobina glucosilada $\left(\mathrm{HbA}_{\mathrm{IC}}\right)$, colesterol total, triglicéridos, tensión arterial, índice de masa corporal e índice cintura/cadera. Se obtuvieron datos sobre edad, sexo, tiempo de evolución, complicaciones diabéticas y comorbilidad. Resultados. Completaron el estudio 249 sujetos de los cuales $62.7 \%$ fueron mujeres. El alfa de Cronbach fue $\geq 0.80$ para los dominios. La consistencia test-retest para la calificación total tuvo una $r=0.82, p=0.01$. La mediana de la puntuación total fue de 29 puntos (escala de 0 a I00) y se tomó como punto de corte para definir mejor $(<29)$ y peor $(\geq 29)$ calidad de vida. En el análisis bivariado, una peor calidad de vida se asoció con complicaciones tardías de la diabetes $\geq$ I $(R M=$ I.73; IC95\% I.05-3.06); colesterol total $>240$ $\mathrm{mg} / \mathrm{dL}(R M=4.43$; IC95\% I.23-I6.26); comorbilidad $\geq 2$ enfermedades $(R M=2.36$; IC95\% I.3I-4.28); evolución mayor a 10 años $\left(R M=2.19\right.$; IC95\% I.27-3.78) y $\mathrm{HbA}_{\mathrm{IC}}>8 \%(R M=$ I.8I; IC95\% I.09-2.99). Las tres últimas fueron predictoras de peor calidad de vida en la regresión logística $(p<0.05)$.
López-Carmona JM, Rodríguez-Moctezuma R.

Adaptation and validation of quality

of life instrument Diabetes 39 for Mexican patients

with type 2 diabetes mellitus.

Salud Publica Mex 2006;48:200-2II.

\section{Abstract}

Objective. To adapt and validate the Spanish language version of the Diabetes 39 instrument, which measures quality of life, for Mexican patients with type 2 diabetes mellitus (DM2). Materials and Methods:The Spanish language version of the instrument was adapted to make it more comprehensible to Mexican patients. In a cross-sectional survey, the instrument was administered on two different days to 260 patients with type DM-2. Glycated hemoglobin $\left(\mathrm{HbA}_{\mathrm{IC}}\right)$, total cholesterol, triglycerides, blood pressure, body mass index and waist/hip ratio were measured. Information about age, sex, time since diagnosis of diabetes, diabetes related complications and comorbidity was obtained. Results. A total of 249 subjects completed the study, $62.7 \%$ of which were women. Cronbach's $\alpha$ coefficient was $\geq 0.80$ for the domains and 0.95 for the total score. The test-retest consistency for the total score was $r=0.82, p=0.01$. The median of the total score was 29 (on a scale of $0-100$ ), which was considered the cutoff value for defining "better" $(<29)$ and "worse" $(\geq 29)$ quality of life. A worse quality of life was associated with diabetes related complications $\geq 1 \quad(O R=1.73 ;$ IC95\% I.05-3.06); total cholesterol $>240 \mathrm{mg} / \mathrm{dL}$ (OR= 4.43; IC95\% I.23-I6.26); comorbidity $\geq 2$ diseases ( $O R=2.36$; IC95\% I.3I -4.28$)$; diabetes longer than 10 years $\left(O R=2.19 ; I C 95 \%\right.$ I.2-3.78), and $\mathrm{HbA}_{\mathrm{IC}}$ $>8 \%(O R=1.81 ;$ IC95\% I.09-2.99). The last three were identified as predictor variables for worse quality of life by logistic

(I) Unidad de Medicina Familiar No. 9I, Instituto Mexicano del Seguro Social (IMSS), Coacalco, Estado de México, México.

(2) Maestro en Investigación Clínica, Módulo de Investigación, Hospital General, Regional 72 Lic. Vicente Santos Guajardo, Delegación Estado de México Oriente, Instituto Mexicano del Seguro Social, México.

Fecha de recibido: 3 I de marzo de 2005 - Fecha de aprobado: 10 de enero de 2006 Solicitud de sobretiros: Juan Manuel López Carmona. Unidad de Medicina Familiar No. 91 Coacalco, José López Portillo 204, col. Zacuautitla, 55700 Coacalco, Estado de México, México. Correo electrónico: jmlopcar@prodigy.net.mx 
Conclusiones. La versión adaptada en idioma español del Diabetes 39 es un instrumento válido y confiable para medir la calidad de vida en los pacientes mexicanos con DM-2.

Palabras clave: calidad de vida; diabetes mellitus; validación; cuestionarios; México regression $(p<0.05)$. Conclusions. The adapted Spanish language version of Diabetes 39 is a reliable and valid instrument for measuring the quality of life of Mexican patients with DM-2.

Key words: quality of life; diabetes mellitus; validation; questionnaires; Mexico
E 1 concepto denominado calidad de vida implica la evaluación global que un sujeto hace de su vida con base en sus características personales (demográficas, valores, personalidad) y en factores externos entre los que se encuentran las enfermedades y el tratamiento que éstas requieren. ${ }^{1-3}$ La calidad de vida relacionada con la salud incluye las esferas física, psicológica y social de la salud vistas como áreas diferentes que están influidas por las experiencias, creencias, expectativas y percepciones de las personas. ${ }^{4}$ Cada esfera del estado de salud puede medirse en dos dimensiones: una evaluación objetiva del funcionamiento y una percepción subjetiva del individuo. ${ }^{5}$ Esta última puede explicar que dos personas con un mismo estado de salud puedan tener muy diferente calidad de vida., ${ }^{4,6}$

La calidad de vida ha sido motivo de interés creciente para los clínicos en las tres últimas décadas debido a que se le considera una variable de desenlace muy importante de la atención médica, y para medirla se han construido instrumentos genéricos y específicos ${ }^{7}$ que varían de acuerdo con el tipo de pacientes en estudio, escenario de administración y tipo de cuestionario (formato breve, autoadministración, entrevista, por teléfono o por correo). ${ }^{3}$ Los instrumentos genéricos se utilizan para evaluar un amplio rango de características aplicables a diversas enfermedades o condiciones; en cambio, los instrumentos específicos tienen un enfoque dirigido a las características más relevantes de la enfermedad o condición en estudio, así como de los pacientes. ${ }^{7}$

Las complicaciones agudas y crónicas de la diabetes mellitus, del mismo modo que su tratamiento -con frecuencia complejo, costoso, difícil de seguir o con efectos secundarios molestos-, pueden impactar la calidad de vida de quienes la padecen. Para medir la calidad de vida en los pacientes con diabetes tipos 1 y 2 se utilizan diversos instrumentos genéricos ${ }^{8-13}$ y específicos ${ }^{9,10,14-17}$ cuya información parece ser complementaria. ${ }^{9-10}$

El cuestionario Diabetes $39^{15}$ se diseñó para medir la calidad de vida en pacientes con diabetes mellitus tipos 1 y 2. Este instrumento fue seleccionado para su validación en México debido a que es multidimensional y cubre aspectos de bienestar psicológico y fun- cionamiento social, entre otros (algunos instrumentos específicos son unidimensionales o se diseñaron para aplicarse exclusivamente en pacientes con alguno de los dos tipos de diabetes); es un cuestionario que se centra en los pacientes diabéticos y en cuya elaboración participaron expertos de diversas disciplinas, así como sujetos con esta enfermedad, además de que es relativamente corto, de autoadministración y durante su construcción demostró tener elevada consistencia interna, así como validez de contenido y criterio (fue comparado con el cuestionario Short Form-36 Health Survey [SF-36], que se considera el instrumento genérico de calidad de vida más relevante para los pacientes con diabetes). ${ }^{15}$ En la actualidad se le estima como uno de los instrumentos específicos con mejor validez para medir la calidad de vida en los pacientes con diabetes ${ }^{18,19}$ y, en comparación con otros instrumentos específicos, las puntuaciones que otorga guardan la más alta correlación con las puntuaciones del SF-36. ${ }^{18} \mathrm{~A}$ pesar de estas características, no se conocen nuevos informes de su uso en poblaciones distintas a la de su validación inicial. También resta por medirse su reproducibilidad test-retest y determinar si se produce alguna correlación entre las calificaciones que otorga y los marcadores de control metabólico u otras variables clínicas. Este instrumento no ha sido validado en población mexicana.

Los objetivos de este estudio fueron a) adaptar la versión en español del instrumento Diabetes 39 para aplicarlo a población mexicana con DM-2; b) evaluar su reproducibilidad test-retest, y c) explorar, como parte de su validación, si existe correspondencia entre la calificación que otorga de la calidad de vida y algunas variables clínicas, bajo la hipótesis de que una peor calidad de vida guardaría relación con un mayor tiempo de evolución de la diabetes, presencia de complicaciones diabéticas, enfermedades concomitantes activas o deficiente control metabólico.

\section{Material y métodos}

Entre julio de 2000 y enero de 2002 se realizó una encuesta transversal en la Unidad de Medicina Familiar 
(UMF) número 91 del Instituto Mexicano del Seguro Social, ubicada en Coacalco, Estado de México, dentro del área conurbana de la Ciudad de México. El Comité Local de Investigación aprobó el protocolo respectivo.

\section{Instrumento}

El instrumento Diabetes 39, en su versión en idioma español hablado en España, fue proporcionado por G. Boyer, ${ }^{15}$ uno de los autores de su versión original en inglés, mediante comunicación personal. Esta versión en español, que no contaba con validación previa, fue adaptada al español hablado en México mediante modificaciones mínimas en la redacción de algunos ítemes, por consenso de un panel de cinco investigadores con experiencia en la construcción y validación de cuestionarios, con el fin de facilitar su comprensión en los pacientes mexicanos con DM-2.

El cuestionario contiene 39 ítemes cerrados que se agrupan en cinco secciones: Energía-Movilidad (15 ítemes: 3, 7, 9, 10, 11, 12, 13, 16, 25, 29, 32, 33, 34, 35 y 36), Control de la diabetes (12 ítemes: 1, 4, 5, 14, 15, 17, 18, 24, 27, 28, 31 y 39); Ansiedad-preocupación (cuatro ítemes: 2, 6, 8 y 22), Carga social (cinco ítemes: 19, 20, 26, 37 y 38) y Funcionamiento sexual (tres ítemes: 21, 23 y 30) (véase el anexo). Los pacientes responden qué tanto está afectada su calidad de vida durante el último mes por la acción o actividad que expresa cada ítem, colocando una $X$ en una escala de tipo análoga visual modificada, que en su versión original se presenta como una línea horizontal continua con marcas verticales que delimitan espacios donde se ubican los números 1 al 7 , cuya escala de valores es el número 1 para nada afectado en lo absoluto y el 7 a sumamente afectado en la calidad de vida. Cuando la $X$ toca alguna marca vertical, la calificación debe redondearse en 0.5 puntos por arriba o por debajo de la puntuación contenida entre las dos marcas próximas al número elegido, por lo que las puntuaciones mínima y máxima para cada respuesta van de 0.5 a 7.5. A continuación, las puntuaciones de cada sección se transforman a una escala de 0 a 100 mediante una fórmula para su conversión lineal. Contiene dos ítemes finales que califican la percepción del paciente acerca de su calidad de vida de manera global, con un rango del 1 (mínima) al 7 (máxima), y la severidad de la diabetes con un rango del 1 (ninguna severidad) al 7 (extremadamente grave). El primer ítem permite medir la percepción que el paciente tiene de su calidad de vida como un todo y determinar si existe correlación entre ésta y la calificación global de la calidad de vida obtenida con el instrumento. El instrumento no contiene una definición de calidad de vida, por lo que el paciente contesta de acuerdo con lo que significa este concepto en su percepción individual. El segundo ítem mide la percepción que el paciente tiene sobre la severidad de su diabetes y permite identificar si hay congruencia de este valor con la calificación de su calidad de vida.

Para el presente estudio, una barra horizontal dividida en celdas que contienen en su interior los números del 1 al 7 sustituyó a la línea, y se asignó la puntuación de la celda donde se ubicó la $X$ sin hacer aproximaciones de 0.5 puntos para simplificar su calificación. La suma de las puntuaciones obtenidas en cada sección, la puntuación total y las calificaciones de autopercepción de la calidad de vida y de la gravedad de la diabetes fueron transformadas a una escala de 0 a 100 con el auxilio de las siguientes fórmulas para su transformación lineal:

- Energía y movilidad:

[(calificación cruda - 15) / (105-15)] x 100

- Control de la diabetes:

[(calificación cruda - 12) / (84-12)] x 100

- Ansiedad-preocupación:

[(calificación cruda - 4) / (28-4)] x 100

- Carga social:

[(calificación cruda - 5) / (35-5)] x 100

- Funcionamiento sexual:

[(calificación cruda - 3) / (21-3)] x 100

- Calificación total:

[(calificación cruda - 39) / (273-39)] x 100

- Calidad de vida:

[(calificación cruda - 1) / (7-1) x 100

- Severidad de la diabetes:

[(calificación cruda - 1) / (7-1) x 100

No se contempla una ponderación especial para la calificación de cada sección en la calificación global más allá de la que representa el número de ítemes de cada una de las mismas, y la puntuación total refleja la afectación en el concepto calidad de vida como un todo.

\section{Pacientes}

Fueron encuestados 260 pacientes adultos con diagnóstico de DM-2 de ambos sexos en tratamiento médico, que supiesen leer y escribir, seleccionados al azar simple en forma diaria entre quienes acudían a su cita de control. Todos los pacientes proporcionaron su consentimiento informado y procedieron de ambos turnos (matutino y vespertino) de los 17 consultorios de medicina familiar con que cuenta la UMF 91, y de ese modo contribuyeron a conformar una muestra representativa de la población de diabéticos adscritos a la clínica. La proporción de los que rechazaron participar en el es- 
tudio fue inferior a 5\%. Se excluyó a pacientes embarazadas o con diabetes tipo 1 . El tamaño de la muestra se calculó a partir de un mínimo de cinco pacientes por cada ítem del instrumento.

\section{Mediciones}

No se realizaron maniobras de intervención terapéutica. Los pacientes respondieron el cuestionario de manera autoadministrada en un aula de la UMF 91 en dos días diferentes, con un intervalo promedio de 21 días (rango de 15 a 35 días).

Se tomaron muestras en ayunas de sangre venosa para medir la $\mathrm{HbA}_{1 \mathrm{C}}$ por inmunoensayo enzimático de micropartículas mediante el equipo IMX System del laboratorio ABBOTT, División ABBOTT Diagnóstico, y colesterol total y triglicéridos por técnica colorimétrica mediante procedimientos manuales. Se pesó y midió a los pacientes sin calzado y con ropa ligera de calle en una báscula calibrada con estadímetro. El perímetro de la cintura se midió con el paciente en bipedestación, con cinta métrica flexible inmediatamente por arriba de las crestas iliacas a la altura de la cicatriz umbilical, y el perímetro de la cadera se midió a la altura del punto más sobresaliente del área glútea, sobre el pubis. Se calculó el índice de masa corporal (IMC) de acuerdo con la fórmula de Quetelet (peso en kilogramos dividido entre el cuadrado de la talla en metros) y se obtuvo el índice cintura/cadera (ICC) al dividir el perímetro de la cintura entre el perímetro de la cadera. La tensión arterial (TA) fue medida con esfigmomanómetro mercurial y estetoscopio de acuerdo con la auscultación de los ruidos de Korotkoff.

De acuerdo con los criterios de la Organización Mundial de la Salud ${ }^{20}$ se consideró que había sobrepeso si el IMC se encontraba entre 25.1 y $30 \mathrm{~kg} / \mathrm{m}^{2}$ y obesidad si era mayor de $30 \mathrm{~kg} / \mathrm{m}^{2}$. La $\mathrm{HbA}_{1 \mathrm{C}^{\prime}}$ el colesterol total, los triglicéridos y la tensión arterial se clasificaron según los puntos de corte que contempla la Norma Oficial Mexicana NOM-015-SSA2-1994, para la prevención, tratamiento y control de la diabetes mellitus. ${ }^{21}$ En esta norma el grado de control metabólico se clasifica en bueno, regular y malo, respectivamente, de acuerdo con los siguientes valores: $\mathrm{HbA}_{1 \mathrm{C}}$ : $<6.5,6.5-8.0 \mathrm{y}>$ de $8.0 \%$; colesterol total: $<200$, 200-239 y $\geq 240 \mathrm{mg} /$ dl; triglicéridos: <150, 150-200 y $>200$ mg/dl; tensión arterial: <120/80, 121-129/81-84 $\mathrm{y} \geq 130 / 85 \mathrm{mmHg}$.

En el expediente médico se buscaron los registros de comorbilidad y complicaciones relacionadas con la diabetes, para considerarlas presentes si los diagnósticos estaban consignados en ese documento, así como los valores de la glucemia en ayuno de los últimos tres meses, realizadas por la técnica de glucosa-oxidasa, tras lo cual se calculó su valor promedio.

Se consideraron como variables independientes el género, el tiempo de evolución, la comorbilidad, las complicaciones tardías de la diabetes y los parámetros de control metabólico $\left(\mathrm{HbA}_{1 \mathrm{C}^{\prime}}\right.$ glucemia promedio, colesterol total, triglicéridos, IMC, ICC, TA). La variable dependiente fue la calidad de vida evaluada con el instrumento Diabetes 39. Se consideró mejor calidad de vida si la puntuación total individual era menor que la mediana grupal, y peor calidad de vida si era igual o mayor.

\section{Análisis estadístico}

Se realizó estadística descriptiva. Se calculó el coeficiente de correlación de Spearman para medir la consistencia test-retest y la correlación de la calificación total con la calidad de vida y con la gravedad de la diabetes. Se determinó la consistencia interna mediante alfa de Cronbach. Se utilizó la prueba U de Mann Whitney para identificar diferencias en las calificaciones por dominio y total según el sexo y la de Kruskal Wallis para diferencias por estratos de edad. Se buscaron diferencias por sexo en los valores de variables clínicas y metabólicas por medio de ji cuadrada. La asociación entre las variables independientes y la calidad de vida se estableció mediante análisis bivariado por el cálculo de razón de momios (RM). Las variables predictoras de peor calidad de vida se identificaron mediante regresión logística en la que se incluyeron simultáneamente las variables que obtuvieron significancia en el análisis bivariado. Se utilizó el paquete estadístico SPSS para Windows.

\section{Resultados}

Completaron el estudio 249 pacientes (95.8\%); 156 $(62.7 \%)$ fueron mujeres. La edad promedio fue de 52.5 años para los varones (rango de 24 a 75 años) y de 55.7 para las mujeres (rango de 34 a 91 años). El tiempo de evolución de la diabetes osciló entre un mes y 38 años,

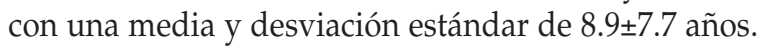
El tiempo para contestar el cuestionario fue de $15 \mathrm{mi}$ nutos en promedio (rango de 7 a 25 minutos) y 91.2\% de los pacientes recibía tratamiento farmacológico. Las características generales de la muestra se presentan en el cuadro I.

La media y desviación estándar de los parámetros de control metabólico fueron: glucosa 189.4 \pm 60.3 $\mathrm{mg} / \mathrm{dl} ; \mathrm{HbA}_{1 \mathrm{C}} 8.2 \pm 1.8 \%$; colesterol $173.6 \pm 41.5 \mathrm{mg} / \mathrm{dl}$; triglicéridos $217.8 \pm 159.0 \mathrm{mg} / \mathrm{dl}$; TAS $132.3 \pm 12.8$ $\mathrm{mmHg}$; TAD $83.7 \pm 7.7 \mathrm{mmHg}$; IMC $30.1 \pm 6.0 \mathrm{~kg} / \mathrm{m}^{2} \mathrm{e}$ 
Cuadro I

Características generales de 249 pacientes Con diabetes tipo 2. Unidad de Medicina Familiar 9I. MÉxico, 2000-2002

\begin{tabular}{lcc} 
& No. & $\%$ \\
$\begin{array}{l}\text { Sexo } \\
\text { Mujeres }\end{array}$ & 156 & 62.5 \\
\hline Hombres & 93 & 37.3 \\
Edad (años) & & \\
$<40$ & 12 & 4.8 \\
\hline 40 a 59 & 151 & 60.7 \\
\hline 60 o más & 86 & 34.5 \\
& & \\
Ocupación \\
Labores del hogar & 133 & 53.4 \\
\hline Trabajador en activo & 83 & 33.4 \\
\hline Pensionado o jubilado & 29 & 11.6 \\
\hline Desempleado & 4 & 1.6 \\
Estado civil & & \\
Soltero & 12 & 4.8 \\
\hline Casado & 192 & 76.9 \\
\hline Viudo & 36 & 14.7 \\
\hline Divorciado & 3 & 1.2 \\
\hline Unión libre & 6 & 2.4
\end{tabular}

Escolaridad (años)

\begin{tabular}{lrr} 
Hasta 6 & 140 & 56.2 \\
\hline 7 a 9 & 61 & 24.6 \\
\hline 10 a I2 & 21 & 8.4 \\
\hline 13 o más & 20 & 8.0 \\
\hline Sin dato & 7 & 2.8
\end{tabular}

Tiempo de evolución (años)

\begin{tabular}{lrl}
$\leq 5$ & 105 & 42.1 \\
\hline 6 a 10 & 66 & 26.5 \\
\hline$>10$ & 78 & 31.4
\end{tabular}

Tratamiento

\begin{tabular}{lrr} 
Dieta solamente & 17 & 6.8 \\
\hline Antidiabéticos orales & 179 & 71.9 \\
\hline Insulina & 42 & 16.9 \\
\hline Antidiabéticos orales + insulina & 6 & 2.4 \\
\hline Sin dato & 5 & 2.0
\end{tabular}

ICC 0.91 \pm 0.09 . Casi tres cuartas partes de los pacientes presentaron $\mathrm{HbA}_{1 \mathrm{C}} \geq 7.0 \%$. Los valores de las variables de control metabólico se muestran en el cuadro II.

Las enfermedades que con más frecuencia se asociaron a la diabetes abarcaron: obesidad, con IMC >30 $\mathrm{kg} / \mathrm{m}^{2}$ en 119 pacientes (47.8\%); hipertensión arterial
$>130 / 85 \mathrm{mmHg}$ en 116 (46.6\%); hipertrigliceridemia $\geq 150 \mathrm{mg} / \mathrm{dl}$ en 151 (60.6\%), e hipercolesterolemia >200 $\mathrm{mg} / \mathrm{dl}$ en $62(24.9 \%)$.

Los pacientes con al menos una complicación tardía de la diabetes registrada en el expediente fueron $86(34.5 \%)$, y de éstas las más frecuentes fueron la retinopatía en $37(14.8 \%)$, nefropatía en 23 (9.2\%), neuropatía periférica o visceral en $21(8.4 \%)$ y cardiopatía

\section{Cuadro II}

VALORES DE LAS VARIABLES CLÍNICAS Y BIOQUímiCAS, SEGÚN SEXO, EN 249 PACIENTES CON DIABETES TIPO 2. Unidad de Medicina Familiar 9I. México, 2000-2002

Hombres $(n=93)$ Mujeres $(n=156) \quad p^{*}$

Índice de masa corporal $\left(\mathrm{kg} / \mathrm{m}^{2}\right)$

\begin{tabular}{llll}
$<25$ & $18(19.3)$ & $19(12.2)$ & 0.01 \\
\hline 25 a 30 & $42(45.2)$ & $51(32.7)$ & \\
\hline$>30$ & $33(35.5)$ & $86(55.1)$ &
\end{tabular}

Índice cintura/cadera

Normal (hasta 0.8 en mujeres

\begin{tabular}{lrrr} 
y 0.9 en hombres) & $19(20.4)$ & $15(9.6)$ & 0.02 \\
\hline Alto & $74(79.6)$ & $141(90.4)$ &
\end{tabular}

Glucemia promedio de tres meses $(\mathrm{mg} / \mathrm{dl})$

\begin{tabular}{lrrr}
$\leq 110$ & $9(9.7)$ & $8(5.1)$ & 0.04 \\
\hline 110 a 139 & $20(21.5)$ & $19(12.2)$ & \\
\hline$\geq 140$ & $64(68.8)$ & $129(82.7)$ &
\end{tabular}

Hemoglobina $A_{I C}(\%)$

\begin{tabular}{llll}
$<6.5$ & $21(22.6)$ & $25(16.0)$ & 0.03 \\
\hline 6.5 a 8.0 & $33(35.5)$ & $38(24.4)$ & \\
\hline$>8.0$ & $39(41.9)$ & $93(59.6)$ &
\end{tabular}

Colesterol total $(\mathrm{mg} / \mathrm{dl})$

\begin{tabular}{lrrr}
$<200$ & $75(80.6)$ & $112(71.8)$ & 0.26 \\
\hline 200 a 239 & $13(14.0)$ & $33(21.2)$ & \\
\hline 2240 & $5(5.4)$ & II $(7.0)$ &
\end{tabular}

Triglicéridos $(\mathrm{mg} / \mathrm{dl})$

\begin{tabular}{llll}
$<150$ & $33(35.5)$ & $65(41.7)$ & 0.37 \\
\hline 150 a 200 & $18(19.3)$ & $35(22.4)$ & \\
\hline$>200$ & $42(45.2)$ & $56(35.9)$ &
\end{tabular}

Tensión arterial $(\mathrm{mmHg})$

\begin{tabular}{llll}
$\leq 120 / 80$ & $25(26.9)$ & $40(25.7)$ & 0.94 \\
\hline$|2|-|30 / 8|-85$ & $26(27.9)$ & $42(26.9)$ & \\
\hline$>130 / 85$ & $42(45.2)$ & $74(47.4)$ &
\end{tabular}

* Prueba ji cuadrada 
isquémica en 17 (6.8\%); sólo 19 (7.6\%) pacientes tuvieron dos o más complicaciones.

Los 39 ítemes del instrumento mostraron una correlación ítem-total mayor de 0.3 , con la mínima de 0.34 para el ítem 4 y la máxima de 0.75 para el ítem 14 . Los coeficientes $\alpha$ de Cronbach por dominio y calificación total fueron: energía y movilidad (15 ítemes), $\alpha=0.92$; control de la diabetes (12 ítemes), $\alpha=0.83$; ansiedadpreocupación (4 ítemes), $\alpha=0.80$; carga social (5 íte$\mathrm{ms}$ ), $\alpha=0.83$; funcionamiento sexual (3 ítemes), $\alpha=0.93$ y calificación total (39 ítemes), $\alpha=0.95$.

La mediana de la calificación total para la muestra fue de 29 puntos (escala de 0 a 100). En el análisis estratificado por sexo, las medianas de las calificaciones, por sección y total, mostraron mayor afectación en las mujeres en la sección energía y movilidad (35 contra 27, $p=0.02$ ), en tanto que los hombres presentaron mayor afectación en el dominio funcionamiento sexual (50 contra 11, $p<0.001)$, como se muestra en el cuadro III.

En el análisis estratificado por edad se observó que presentan mayor ansiedad y preocupación por la diabetes los pacientes $<60$ años que los mayores de esta edad (medianas de 42 contra 33, $p<0.01$ ), en tanto que los menores de 70 años califican con mayor afectación en el dominio Funcionamiento sexual que los mayores de esa edad (medianas de 33 contra $1, p<0.01$ ). No hubo diferencias significativas por grupos de edad en otras secciones ni en la calificación total.

$\mathrm{Al}$ estratificar por tiempo de evolución, los sujetos con cinco años o menos desde el diagnóstico tienen puntuación más baja (menor afectación en su calidad de vida) que los de 6 a 10 o más de 10 años, con diferencias significativas en las secciones control de la diabetes, carga social, función sexual y energía y movilidad, así como en la calificación total (medianas de 23,33 y 34 respectivamente, $p=0.003$ ), pero no se encontraron diferencias en la sección ansiedad-preocupación (medianas de 42 para los tres grupos, $p=0.46$ ). Los pacientes con evolución hasta de cinco años también percibieron como menos grave su diabetes en comparación con los otros dos grupos (medianas de 33, 50 y 58 respectivamente, $p=0.036$ ).

La correlación test-retest fue buena para todas las secciones y la calificación total. Se encontró correlación negativa entre las cinco secciones del instrumento y la calidad de vida percibida por el paciente, así como correlación positiva entre las calificaciones del instrumento y la gravedad de la diabetes (cuadro IV).

Las variables que se vincularon con peor calidad de vida ( $\geq 29$ puntos) en el análisis bivariado fueron presencia de una o más complicaciones tardías, dos o más enfermedades asociadas, evolución de la diabetes

\section{Cuadro III \\ Medianas, Percentil 25 y percentil 75, por sección Y CALIFICACIÓN TOTAL (0 A I 00 PUNTOS) del instrumento Diabetes 39, Según sexo, EN 249 PACIENTES CON DIABETES TIPO 2}

\begin{tabular}{|c|c|c|c|c|}
\hline \multirow{2}{*}{$\begin{array}{l}\text { Sección } \\
\text { (número de ítemes) }\end{array}$} & \multirow{2}{*}{$\begin{array}{l}\text { Mediana muestral } \\
\quad(p 25-p 75)\end{array}$} & \multicolumn{2}{|c|}{$\begin{array}{l}\text { Mediana por sexo } \\
\quad(p 25-p 75)\end{array}$} & \multirow[b]{2}{*}{$p^{*}$} \\
\hline & & Mujeres & Hombres & \\
\hline Control de la diabetes & 22 & 26 & 19 & 0.13 \\
\hline (12 ítemes) & $(I I-38)$ & $(|2-4|)$ & $(10-33)$ & \\
\hline Ansiedad/preocupación & 42 & 46 & 38 & 0.10 \\
\hline (4 ítemes) & $(2 \mid-58)$ & $(21-58)$ & $(17-54)$ & \\
\hline Carga social & 17 & 17 & 17 & 0.47 \\
\hline (5 ítemes) & $(7-37)$ & $(7-42)$ & $(3-30)$ & \\
\hline
\end{tabular}

\begin{tabular}{lcccc} 
Funcionamiento sexual & 28 & II & 50 & $<0.00 \mathrm{I}$ \\
\hline$(3$ itemes $)$ & $(0-72)$ & $(0-6 \mathrm{I})$ & $(25-83)$
\end{tabular}

\begin{tabular}{lcccc} 
Energía y movilidad & 30 & 35 & 27 & 0.02 \\
\hline (15 ítemes) & $(16-50)$ & $(19-52)$ & $(9-45)$ &
\end{tabular}

\begin{tabular}{lcccc} 
Calificación total & 29 & 30 & 28 & 0.25 \\
\hline (39 ítemes) & $(16-46)$ & $(18-48)$ & $(12-39)$ &
\end{tabular}

\begin{tabular}{lcccc} 
Calidad de vida & 50 & 50 & 50 & 0.60 \\
\hline (I item) & $(33-83)$ & $(33-83)$ & $(33-75)$ &
\end{tabular}

\begin{tabular}{lcccc} 
Severidad de la diabetes & 50 & 50 & 50 & 0.85 \\
\hline$($ I ítem $)$ & $(17-83)$ & $(17-83)$ & $(17-79)$ &
\end{tabular}

*U de Mann Whitney

mayor de 10 años, $\mathrm{HbA}_{1 \mathrm{C}}>8 \%$ y colesterol $>240 \mathrm{mg} / \mathrm{dl}$ (cuadro V). El modelo multivariado de regresión logística identificó como variables predictoras de peor calidad de vida a la presencia de dos o más enfermedades concomitantes ( $R M=2.31$; IC95\% 1.25-4.26); evolución mayor de 10 años ( $R M=1.90$; IC95\% 1.08-3.35), y $\mathrm{HbA}_{1 \mathrm{C}}>8 \%(R M=1.75$; IC95\% 1.03-2.96); todas con $p<0.05$.

\section{Discusión}

Los resultados muestran que la versión adaptada al español que se habla en México del instrumento de calidad de vida Diabetes 39 tiene un alto nivel de consistencia interna y test-retest, así como relaciones lógicas entre las calificaciones que otorga y los valores de 


\section{Cuadro IV \\ Coeficientes de correlación del instrumento Diabetes 39 (TEST-Retest y De los dominios CON LA CALIFICACIÓN TOTAL, CALIDAD DE VIDA Y SEVERIDAD DE LA DIABETES) EN 249 PACIENTES MEXICANOS CON DIABETES TIPO 2}

Test-retest

Control de la diabetes ( 12 ítemes)

Ansiedad-preocupación (4 ítemes)

Carga social (5 ítemes)

Función sexual (3 ítemes)

Energía movilidad (I5 ítemes)

Calificación total (39 ítemes)

Calidad de vida (I ítem)

Severidad de la diabetes (I ítem)

Correlación de Spearman, con $p=0.01$ para todos los coeficientes
Calidad de vida

Severidad de la diabetes

\begin{tabular}{lccc}
0.79 & 0.88 & -0.32 & 0.61 \\
\hline 0.78 & 0.76 & -0.28 & 0.42 \\
\hline 0.77 & 0.83 & -0.36 & 0.58 \\
\hline 0.84 & 0.51 & -0.24 & 0.30 \\
\hline 0.84 & 0.92 & -0.32 & 0.49 \\
\hline 0.82 & 1.0 & -0.35 & 0.60 \\
\hline 0.58 & -0.35 & 1.0 & -0.24 \\
0.71 & 0.60 & -0.24 & 1.0
\end{tabular}

Cuadro V

FuERZA DE ASOCIACIÓN EN EL ANÁLISIS BIVARIADO ENTRE LAS VARIABLES CLÍNICAS Y PEOR CALIDAD DE VIDA (CALIFICACIONES $\geq$ MEDIANA POR DOMINIO Y TOTAL) EN 249 PACIENTES MEXICANOS CON DiABETES TIPO 2. RAZÓN de MOMIOS (IC95\%)

\begin{tabular}{|c|c|c|c|c|c|c|}
\hline & Control de la diabetes & Ansiedad-preocupación & Carga social & Función sexual & Energía-movilidad & Total \\
\hline \multirow[t]{2}{*}{$\mathrm{HbA}_{I \mathrm{C}}>8 \%$} & 2.36 & 1.28 & I.78। & 1.18 & 1.22 & 1.81 \\
\hline & $(1.42-3.94)$ & $(0.78-2 . I I)$ & $(1.07-2.95)$ & $(0.72-1.95)$ & $(0.72-2.01)$ & $(1.09-2.99)$ \\
\hline \multirow[t]{2}{*}{ Colesterol $>240 \mathrm{mg}$} & 1.59 & 2.44 & 1.35 & 2.20 & 2.55 & 4.43 \\
\hline & $(0.55-4.6 I)$ & $(0.8 \mid-7.35)$ & $(0.47-3.83)$ & $(0.73-6.63)$ & $(1.15-5.64)$ & $(1.23-16.26)$ \\
\hline
\end{tabular}

\begin{tabular}{lcccccc} 
Tiempo de evolución $>10$ años & 1.88 & 1.08 & 1.22 & 1.65 & 1.88 & 2.192 \\
\hline & $(1.09-3.22)$ & $(0.63-1.84)$ & $(0.72-2.08)$ & $(0.97-2.83)$ & $(1.09-3.22)$ & $(1.27-3.78)$
\end{tabular}

\begin{tabular}{ccccccc} 
Complicaciones diabéticas $\geq 1$ & 1.34 & 0.91 & 1.83 & 1.47 & 2.08 & 1.73 \\
\hline & $(0.79-2.27)$ & $(0.53-1.54)$ & $(1.08-3.13)$ & $(0.87-2.50)$ & $(1.21-3.56)$ & $(1.05-3.06)$
\end{tabular}

Enfermedades asociadas $\geq 2$

\begin{tabular}{cccccc}
1.52 & 1.40 & 1.62 & 1.69 & 3.83 & 2.36 \\
\hline$(0.85-2.70)$ & $(0.79-2.47)$ & $(0.91-2.88)$ & $(0.95-3.02)$ & $(2.04-7.18)$ & $(1.31-4.28)$
\end{tabular}

algunas variables de importancia clínica en los sujetos con diabetes tipo 2, lo cual apoya su validez de constructo.

La mediana de la calificación de 29 puntos (escala de 0-100) refleja que la calidad de vida en el grupo estudiado se afectó poco, lo cual es congruente con el hecho de que sólo un tercio de los pacientes presentó complicaciones tardías de la diabetes, ya que la media del tiempo transcurrido desde el diagnóstico fue menor de 10 años. Como era de esperarse, se encontró una alta frecuencia de obesidad, hipertensión y disli- pidemia, los cuales son componentes del síndrome metabólico, presente en muchos de los pacientes con DM-2. Más de 70\% de los sujetos participantes en este estudio estaba en tratamiento con antidiabéticos orales y menos de $20 \%$ recibía insulina, en contraste con los pacientes estudiados durante la validación inicial del instrumento, que en su mayoría se encontraban en tratamiento con insulina. ${ }^{15}$

La consistencia interna de la versión adaptada al español del Diabetes 39 fue alta, con valores alfa de Cronbach $>0.8$ para las secciones y la puntuación total 
(arriba del valor de 0.7 considerado el mínimo aceptable para un buen nivel de consistencia ${ }^{22}$ ) y fueron muy similares a los que se publicaron de su versión original en inglés. ${ }^{15}$

La consistencia test-retest fue buena para las secciones, para la calificación total y para el ítem que explora la severidad de la diabetes, todos ellos con valores de $r$ $>0.70$. El ítem que explora calidad de vida tuvo una $r=$ 0.59 , lo cual puede deberse a que su escala de calificación está invertida en relación con el resto del instrumento, lo que puede causar confusión al responderlo.

No hay informes previos de la correlación testretest, por lo que no hay manera de comparar los resultados obtenidos en el presente estudio. En cambio, las correlaciones positivas entre las calificaciones por secciones y total con la calificación de la severidad de la diabetes, así como la correlación negativa entre las mismas con la calidad de vida, todas con significancia estadística, ya se reportaron antes. ${ }^{15}$

En cuanto al análisis por sexo, los resultados muestran que los hombres presentan mayor afectación en su funcionamiento sexual, lo que coincide con lo que comunicaron Boyer y Earp ${ }^{15}$ durante el desarrollo del Diabetes 39. Aunque en este trabajo no se investigó la presencia de disfunción eréctil, se sabe que representa una complicación frecuente en los hombres con diabetes y que implica peor calidad de vida. ${ }^{23}$ Por otra parte, la sección que reflejó mayor afectación en las mujeres de la muestra fue la de Energía y movilidad, lo cual pudiera estar relacionado con una mayor frecuencia y severidad de la obesidad.

El análisis por tiempo de evolución mostró otras relaciones lógicas en los resultados: existe menor afectación en la calidad de vida de los sujetos con cinco o menos años desde el diagnóstico, y también una percepción de menor gravedad de su diabetes, en comparación con aquellos con conocimiento de su enfermedad de entre 6 y 10 años o más de 10 años. Sin embargo, la única sección sin diferencias fue la de ansiedad-preocupación, lo cual podría indicar que el diagnóstico de diabetes genera una carga de ansiedad permanente en quien la padece.

La asociación entre calidad de vida y control glucémico ha sido motivo de estudios previos tanto transversales como longitudinales, con resultados contradictorios; $6,6,8,13,24,25$ en cambio, la relación entre calidad de vida y presencia de complicaciones tardías de la diabetes ${ }^{9,11,13,24,25}$ o presencia de comorbilidad ${ }^{11,13,15}$ ha sido más consistente.

La relación encontrada en el estudio presente entre peor calidad de vida y descontrol glucémico, mayor tiempo de evolución, presencia de complicaciones tardías y comorbilidad asociada coinciden con el informe de García Peña y colaboradores, ${ }^{13}$ en el que utilizaron el instrumento genérico Sickness Impact Profile para medir la calidad de vida en una muestra de pacientes mexicanos con DM-2; sin embargo, en dicho estudio no se midieron los valores de colesterol. La asociación que encontraron los autores de la presente investigación en el análisis bivariado entre peor calidad de vida y colesterol total $>240 \mathrm{mg}$ tiene sentido lógico pues la hipercolesterolemia favorece la ateroesclerosis, la cual se acelera en los pacientes con diabetes y constituye el fondo de las complicaciones macrovasculares tardías de la diabetes.

Es importante reconocer que el vínculo entre calidad de vida y algunas de las variables clínicas estudiadas es complejo y no necesariamente lineal, lo cual permite contemplar otras hipótesis alternas pues los pacientes que no aceptan introducir cambios en su estilo de vida como realizar ejercicio o adherirse al plan alimentario podrían tener mejor calidad de vida pero peor control metabólico.

Algunas limitaciones de este estudio son que los pacientes se seleccionaron de una sola unidad de medicina familiar y únicamente entre quienes acuden a consulta, lo cual puede ocasionar un sesgo de selección; la población participante estuvo formada por pacientes que saben leer, escribir y son capaces de comprender el instrumento (lo cual es un requisito para la aplicación de instrumentos de autoadministración); la información sobre complicaciones diabéticas y de comorbilidad estuvo basada en los registros de expedientes y no se determinó su severidad, lo cual también puede originar sesgos. Otro factor por considerar es que el estudio fue de tipo transversal, lo que impide efectuar inferencias causales, por lo que se requieren nuevos estudios longitudinales para corroborar los hallazgos encontrados, así como para definir la sensibilidad al cambio del instrumento Diabetes 39 que permita identificar modificaciones en la calidad de vida en estos pacientes, sea por la evolución propia del padecimiento o bien como resultado de maniobras específicas de intervención. Sin embargo, tampoco existen informes sobre este tipo de validación en la mayoría de los demás instrumentos específicos para medir calidad de vida en los sujetos con diabetes. ${ }^{18}$ Además, es importante señalar que los pacientes incluidos en este trabajo muestran un grado importante de descontrol metabólico, lo cual podría reflejar el estado actual de estos pacientes en el sistema nacional de salud, lo que obliga a reflexionar sobre la necesidad de mejorar la calidad de la atención médica en estos enfermos con el fin de mejorar su calidad de vida.

Los resultados obtenidos permiten concluir que la versión en español del instrumento Diabetes 39, 
adaptada en este trabajo, tiene validez y consistencia adecuada para medir la calidad de vida en los pacientes mexicanos con DM-2 que tengan características similares a los incluidos en la presente investigación.

\section{Agradecimientos}

Este trabajo fue realizado parcialmente con apoyo financiero FP-0038/808 otorgado por la Coordinación de Investigación Médica del Instituto Mexicano del Seguro Social, a través del Fondo para el Fomento de la Investigación (FOFOI).

Agradecemos al personal del laboratorio clínico de la Unidad de Medicina Familiar No. 91 por su valiosa ayuda para la realización de las pruebas de laboratorio y a los autores del instrumento Diabetes 39 por habernos facilitado su versión en idioma español.

\section{Bibliografía}

I. Katz S. The science of quality of life. J Chronic Dis 1987;40:459-463. 2. Bergner M. Quality of life, health status and clinical research. Med Care 1989;27 suppl 3:SI48-SI56.

3. Lara-Muñoz MC, Ponce de León S, De la Fuente JR.

Conceptualización y medición de la calidad de vida de pacientes con cáncer. Rev Invest Clín 1995;47:3 I5-327.

4. Testa MA, Simonson DC. Current concepts: assessment of quality-oflife outcomes. N Engl J Med 1996;334(I3):835-840.

5. Muldoon MF, Barger SD, Flory JD, Manuck SB. What are quality of life measurements measuring? BMJ 1998;316(7/30):542-545.

6. Testa MA, Simonson DC, Turner RR.Valuing quality of life and improvements in glycemic control in people with type 2 diabetes. Diabetes Care 1998;2I suppl 3:C44-C52.

7. Guyatt GH,Veldhuyzen Van Zanten SJ, Feeny DH, Patrick DL. Measuring quality of life in clinical trials: a taxonomy and review. CMAJ 1989; | 40: |44|-|448.

8. Wienberger M, Kirkman S, Samsa G, Cowper PA, Shortliffe EA, Simel $\mathrm{DL}$, et al. The relationship between glycemic control and health-related quality of life in patients with non insulin dependent diabetes mellitus. Med Care 1994;32:1 I73-1 I8I.

9. Jacobson AM, de Groot M, Samson JA. The evaluation of two measures of quality of life in patients with type I and type II diabetes. Diabetes Care 1994;17:267-274.
10. Anderson RM, Fitzgerald JT, Kimberlydawn W, Davis WK, Hiss RG. A comparison of global versus disease-specific quality of life measures in patients with NIDDM. Diabetes Care 1997;20:299-305.

II. Glasgow RE, Ruggiero L, Eakin EG, Dryfoos J, Chobanian L. Quality of life and associated characteristics in a large national sample of adults with diabetes. Diabetes Care 1997;20:562-567.

12. Bergner M, Bobbit RA, Carter WB, Gilson BS. The sickness impact profile. Development and final revision of a health status measure. Med Care 1981;19:787-805.

13. García-Peña MC, Reyes-Morales H, Garduño-Espinosa J,

Fajardo-Gutiérrez A, Martínez-García C. La calidad de vida en el paciente diabético tipo Il y factores relacionados. Rev Med IMSS 1995;33:293-298. 14. DCCT Research Group: Reliability and validity of a diabetes quality of life measure for the Diabetes Control and Complications Trial (DCCT). Diabetes Care 1988; I :725-732.

15. Boyer JG, Earp JAL. The development of an instrument for assessing the quality of life of people with diabetes. Med Care 1997;35:440-453. I6. Bott U, Muhlhauser I, Overmann H, Berger M.Validation of a Diabetes-Specific-Quality of Life Scale for patients with type I diabetes. Diabetes Care 1998;21:757-769.

17. Shen W, Kotsanos JG, Huster W, Mathias S, Andrejasich CM, Patrick DL. Development and validation of the Diabetes Quality of Life Clinical Trial Questionnaire. Med Care 1999;37 suppl 4:AS45-AS66.

18. Garrat AM, Schmidt L, Fitzpatrick R. Patient-assessed health outcome measures for diabetes: a structured review. Diabet Med 2002;19:1-II.

19. Watkins K, Connel CM. Measurement of health-related $\mathrm{QOL}$ in diabetes mellitus. Pharmacoeconomics 2004;22(I7): I 109-I I 26.

20. Obesity: preventing and managing the global epidemic. Report of a WHO consultation on obesity. World Health Organ Tech Rep Ser 2000;894:I-XII, I-253.

21. Norma Oficial Mexicana NOM-015-SSA2-1994, para la prevención, tratamiento y control de la diabetes mellitus. México: Secretaría de Salud, 2000.

22. Streiner DL, Norman GR. Health measurement scales. A practical guide to their development and use. 2a. ed. Nueva York: Oxford University Press, 1995.

23. Penson DF, Latini DM, Lubeck DP, Wallace KL, Henning JM, Lue TF. Do impotent men with diabetes have more severe erectile dysfunction and worse quality of life than the general population of impotent patients? Diabetes Care 2003;26:1093-1099.

24. U.K. Prospective Diabetes Study Group. Quality of life in type 2 diabetic patients is affected by complications but not by intensive policies to improve blood glucose or blood pressure control (UKPDS 37). Diabetes Care 1999;22:I I25-II36.

25. Mata CM, Roset GM, Badia LX, Antonanzas VF, Ragel AJ. Impacto de la diabetes mellitus tipo 2 en la calidad de vida de pacientes tratados en las consultas de atención primaria en España. Aten Primaria 2003;31:493-499. 
Anexo

\section{Cuestionario sobre calidad de vida Diabetes 39}

La calidad de vida de las personas está afectada por muchas causas. Estas causas pueden incluir el estado de salud, la oportunidad para vacacionar o divertirse, los amigos, la familia o el trabajo. El siguiente cuestionario se diseñó para ayudar a conocer lo que afecta la calidad de vida en las personas con diabetes.

Las siguientes preguntas se relacionan con el grado de afectación que la diabetes le ocasionó en su calidad de vida durante el último mes. Se le agradecerá que lea cuidadosamente las siguientes preguntas y conteste colocando una cruz $(X)$ en el cuadro del número que refleje mejor el grado de afectación en su vida respecto a cada una de las preguntas señaladas, tomando en cuenta que el número I indica falta de afectación y, al avanzar la numeración, aumenta el grado de afectación en forma progresiva hasta llegar al máximo, que es el número 7, que indica afectación extrema. Si tiene alguna duda, con gusto se le prestará ayuda.

Se le suplica responder todas las preguntas.

Durante el último mes, ¿en qué medida se vio afectada la calidad de su vida por las siguientes causas?

I. El horario de los medicamentos para su diabetes

Nada afectada en absoluto

\begin{tabular}{|l|l|l|l|l|l|l|}
\hline I & 2 & 3 & 4 & 5 & 6 & 7 \\
\hline
\end{tabular}

2. Preocupaciones por problemas económicos

Nada afectada en absoluto

\begin{tabular}{|l|l|l|l|l|l|l|}
\hline I & 2 & 3 & 4 & 5 & 6 & 7 \\
\hline
\end{tabular}

3. Limitación en su nivel de energía

Nada afectada en absoluto

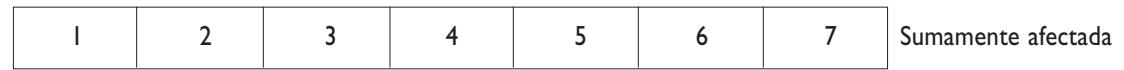

4. Seguir el plan indicado por su médico para el tratamiento de la diabetes

Nada afectada en absoluto

\begin{tabular}{|l|l|l|l|l|l|l|}
\hline I & 2 & 3 & 4 & 5 & 6 & 7 \\
\hline
\end{tabular}

5. No comer ciertos alimentos para poder controlar su diabetes

Nada afectada en absoluto

\begin{tabular}{|l|l|l|l|l|l|l|}
\hline 1 & 2 & 3 & 4 & 5 & 6 & 7 \\
\hline
\end{tabular}

Sumamente afectada

6. Estar preocupado(a) por su futuro

Nada afectada en absoluto

\begin{tabular}{|l|l|l|l|l|l|l|}
\hline I & 2 & 3 & 4 & 5 & 6 & 7 \\
Sumamente afectada
\end{tabular}

7. Otros problemas de salud aparte de la diabetes

Nada afectada en absoluto

\begin{tabular}{|l|l|l|l|l|l|l|}
\hline I & 2 & 3 & 4 & 5 & 6 & 7 \\
\hline
\end{tabular}

8. Tensiones o presiones en su vida

Nada afectada en absoluto

\begin{tabular}{|l|l|l|l|l|l|l|}
\hline I & 2 & 3 & 4 & 5 & 6 & 7 \\
\hline
\end{tabular}

9. Sensación de debilidad

Nada afectada en absoluto

\begin{tabular}{|l|l|l|l|l|l|l|}
\hline I & 2 & 3 & 4 & 5 & 6 & 7 \\
\hline
\end{tabular}

10. Restricciones sobre la distancia que puede caminar

Nada afectada en absoluto

\begin{tabular}{|l|l|l|l|l|l|l|}
\hline 1 & 2 & 3 & 4 & 5 & 6 & 7 \\
\hline
\end{tabular}

II. Los ejercicios diarios que ha de hacer por su diabetes

Nada afectada en absoluto

\begin{tabular}{|l|l|l|l|l|l|l|}
\hline I & 2 & 3 & 4 & 5 & 6 & 7 \\
\hline
\end{tabular}

12. Visión borrosa o pérdida de la visión

Nada afectada en absoluto

\begin{tabular}{|l|l|l|l|l|l|l|}
\hline 1 & 2 & 3 & 4 & 5 & 6 & 7 \\
\hline
\end{tabular}

13. No poder hacer lo que quisiera

Nada afectada en absoluto

\begin{tabular}{|l|l|l|l|l|l|l|}
\hline 1 & 2 & 3 & 4 & 5 & 6 & 7 \\
\hline
\end{tabular}


Durante el último mes, ¿en qué medida se vio afectada la calidad de su vida por las siguientes causas?

14. Tener diabetes

Nada afectada en absoluto

\begin{tabular}{|l|l|l|l|l|l|l|}
\hline 1 & 2 & 3 & 4 & 5 & 6 & 7 \\
Sumamente afectada
\end{tabular}

15. El descontrol de su azúcar en sangre

Nada afectada en absoluto

\begin{tabular}{|l|l|l|l|l|l|l|}
\hline 1 & 2 & 3 & 4 & 5 & 6 & 7 \\
\hline
\end{tabular}

16. Otras enfermedades aparte de la diabetes

Nada afectada en absoluto

\begin{tabular}{|l|l|l|l|l|l|l|}
\hline 1 & 2 & 3 & 4 & 5 & 6 & 7 \\
Sumamente afectada
\end{tabular}

17. Hacerse análisis para comprobar sus niveles de azúcar en sangre

Nada afectada en absoluto

\begin{tabular}{|l|l|l|l|l|l|l|}
\hline 1 & 2 & 3 & 4 & 5 & 6 & 7 \\
\hline
\end{tabular}

18. El tiempo requerido para controlar su diabetes

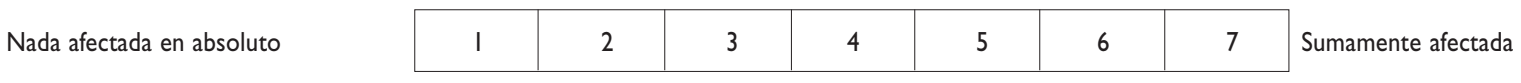

19. Las restricciones que su diabetes impone a su familia y amigos

Nada afectada en absoluto

\begin{tabular}{|l|l|l|l|l|l|l|}
\hline 1 & 2 & 3 & 4 & 5 & 6 & 7 \\
\hline
\end{tabular}

20. La vergüenza producida por tener diabetes

Nada afectada en absoluto

\begin{tabular}{|l|l|l|l|l|l|l|}
\hline $\mathrm{I}$ & 2 & 3 & 4 & 5 & 6 & 7 \\
\hline
\end{tabular}

21. La interferencia de su diabetes en su vida sexual

Nada afectada en absoluto

\begin{tabular}{|l|l|l|l|l|l|l|}
\hline 1 & 2 & 3 & 4 & 5 & 6 & 7 \\
\hline
\end{tabular}

22. Sentirse triste o deprimido

Nada afectada en absoluto

\begin{tabular}{|l|l|l|l|l|l|l|}
\hline 1 & 2 & 3 & 4 & 5 & 6 & 7 \\
Sumamente afectada
\end{tabular}

23. Problemas con respecto a su capacidad sexual

Nada afectada en absoluto

\begin{tabular}{|l|l|l|l|l|l|l|}
\hline 1 & 2 & 3 & 4 & 5 & 6 & 7 \\
\hline
\end{tabular}

24. Tener bien controlada su diabetes

Nada afectada en absoluto

\begin{tabular}{|l|l|l|l|l|l|l|}
\hline 1 & 2 & 3 & 4 & 5 & 6 & 7 \\
\hline
\end{tabular}

25. Complicaciones debidas a su diabetes

Nada afectada en absoluto

\begin{tabular}{|l|l|l|l|l|l|l|}
\hline 1 & 2 & 3 & 4 & 5 & 6 & 7 \\
\hline
\end{tabular}

26. Hacer cosas que su familia y amigos no hacen

Nada afectada en absoluto

\begin{tabular}{|l|l|l|l|l|l|l|}
\hline 1 & 2 & 3 & 4 & 5 & 6 & 7 \\
Sumamente afectada
\end{tabular}

27. Tener que anotar sus niveles de azúcar en sangre

Nada afectada en absoluto

\begin{tabular}{|l|l|l|l|l|l|l|}
\hline 1 & 2 & 3 & 4 & 5 & 6 & 7 \\
\hline
\end{tabular}

28. La necesidad de tener que comer a intervalos regulares

Nada afectada en absoluto

\begin{tabular}{|l|l|l|l|l|l|l|}
\hline I & 2 & 3 & 4 & 5 & 6 & 7 \\
\hline
\end{tabular}

Sumamente afectada

29. No poder realizar labores domésticas u otros trabajos relacionados con la casa

Nada afectada en absoluto

\begin{tabular}{|l|l|l|l|l|l|l|}
\hline I & 2 & 3 & 4 & 5 & 6 & 7 \\
\hline
\end{tabular}

30. Menor interés en su vida sexual

Nada afectada en absoluto

\begin{tabular}{|l|l|l|l|l|l|l|}
\hline $\mathrm{I}$ & 2 & 3 & 4 & 5 & 6 & 7 \\
\hline
\end{tabular}


Durante el último mes, ¿en qué medida se vio afectada la calidad de su vida por las siguientes causas?

3I. Tener que organizar su vida cotidiana alrededor de la diabetes

Nada afectada en absoluto

\begin{tabular}{|l|l|l|l|l|l|l|}
\hline 1 & 2 & 3 & 4 & 5 & 6 & 7 \\
\hline
\end{tabular}

32. Tener que descansar a menudo

Nada afectada en absoluto

\begin{tabular}{|l|l|l|l|l|l|l|}
\hline 1 & 2 & 3 & 4 & 5 & 6 & 7 \\
\hline
\end{tabular}

33. Problemas al subir escaleras

Nada afectada en absoluto

\begin{tabular}{|l|l|l|l|l|l|l|}
\hline 1 & 2 & 3 & 4 & 5 & 6 & 7 \\
\hline
\end{tabular}

34. Dificultades para sus cuidados personales (bañarse, vestirse o usar el sanitario)

Nada afectada en absoluto

\begin{tabular}{|l|l|l|l|l|l|l|}
\hline $\mathrm{I}$ & 2 & 3 & 4 & 5 & 6 & 7 \\
\hline
\end{tabular}

35. Tener el sueño intranquilo

Nada afectada en absoluto

\begin{tabular}{|l|l|l|l|l|l|l|}
\hline 1 & 2 & 3 & 4 & 5 & 6 & 7 \\
\hline
\end{tabular}

36. Andar más despacio que otras personas

Nada afectada en absoluto

\begin{tabular}{|l|l|l|l|l|l|l|}
\hline 1 & 2 & 3 & 4 & 5 & 6 & 7 \\
\hline
\end{tabular}

37. Ser identificado como diabético

Nada afectada en absoluto

\begin{tabular}{|l|l|l|l|l|l|l|}
\hline $\mathrm{I}$ & 2 & 3 & 4 & 5 & 6 & 7 \\
\hline
\end{tabular}

38. Interferencia de la diabetes con su vida familiar

Nada afectada en absoluto

\begin{tabular}{|l|l|l|l|l|l|l|}
\hline 1 & 2 & 3 & 4 & 5 & 6 & 7 \\
\hline
\end{tabular}

39. La diabetes en general

Nada afectada en absoluto

\begin{tabular}{|l|l|l|l|l|l|l|}
\hline 1 & 2 & 3 & 4 & 5 & 6 & 7 \\
\hline
\end{tabular}

Calificación global

I. Por favor, marque con una cruz $(X)$ el cuadro que indique la calificación de su calidad de vida

Mínima calidad

\begin{tabular}{|l|l|l|l|l|l|l|}
\hline $\mathrm{I}$ & 2 & 3 & 4 & 5 & 6 & 7 \\
\hline
\end{tabular}

Máxima calidad

2. Por favor, marque con una cruz $(X)$ el cuadro que indique lo que usted piensa de la gravedad de su diabetes

Ninguna gravedad

\begin{tabular}{|l|l|l|l|l|l|l|}
\hline $\mathrm{I}$ & 2 & 3 & 4 & 5 & 6 & 7 \\
\hline
\end{tabular}

Extremadamente grave

Gracias por sus respuestas 